\title{
Redesign of a University Hospital Preanesthesia Evaluation Clinic using a Queuing Theory Approach
}

\author{
Maartje E. Zonderland, MSc ${ }^{a, b}$, Fred Boer, MD, PhD ${ }^{c, d}$, Richard J. Boucherie, PhD $^{b}$, \\ Annemiek de Roode, MD, PhD ${ }^{d}$, Jack W. van Kleef, MD, PhD $^{d}$
}

${ }^{\text {a} D i v i s i o n ~ I, ~ L e i d e n ~ U n i v e r s i t y ~ M e d i c a l ~ C e n t e r, ~ L e i d e n, ~ t h e ~ N e t h e r l a n d s ~}$

${ }^{b}$ Department of Applied Mathematics, University of Twente, Enschede, the Netherlands

${ }^{\mathrm{C} O R}$ Center, Leiden University Medical Center, Leiden, the Netherlands

${ }^{\mathrm{d} D e p a r t m e n t ~ o f ~ A n e s t h e s i o l o g y, ~ L e i d e n ~ U n i v e r s i t y ~ M e d i c a l ~ C e n t e r, ~ L e i d e n, ~ t h e ~ N e t h e r l a n d s ~}$

\section{Corresponding author:}

Maartje Zonderland

Division I, Leiden University Medical Center

Postbox 9600, 2300 RC Leiden, the Netherlands

m.e.zonderland@lumc.nl 


\begin{abstract}
Background

Changes in patient length of stay (the duration of one visit) as a result of the introduction of an electronic patient file system forced an anesthesia department to change their outpatient clinic organization. The aim of this study is to demonstrate how the involvement of essential employees combined with mathematical techniques to support the decision making process resulted in a successful intervention.
\end{abstract}

\title{
Methods
}

The setting is the preanesthesia evaluation clinic of a university hospital, where patients consult several medical professionals, either on walk-in or appointment basis. Queuing theory was used to model the initial set-up of the clinic, and later to model possible alternative designs. With the queuing model, possible improvements in efficiency could be investigated. Inputs to the model were patient arrival rates and expected service times, collected from the clinic's logging system and by observation. The performance measures calculated with the model were patient length of stay and employee utilization rate. Supported by the model outcomes, a working group consisting of representatives of all clinic employees decided if the initial design should be maintained, or an intervention was needed.

\section{Results}

The queuing model predicted that three of the proposed alternatives would result in better performance. Key points in the intervention were the rescheduling of appointments and the reallocation of tasks. The intervention resulted in a shortening of the time the anesthesiologist needed to decide upon approving the patient for surgery. Patient arrivals increased sharply over one year by more than $16 \%$, however patient length of stay at the clinic remained essentially unchanged. If the initial set-up of the clinic would have been maintained, the patient length of stay would have increased dramatically.

\section{Conclusions}


Queuing theory provides robust methods to evaluate alternative designs for the organization of preanesthesia evaluation clinics. Combining these mathematical techniques with the essential involvement of employees may lead to a successful intervention that improves clinic performance. 


\section{Text}

\section{Introduction}

In the past two decades, it has become common practice to provide preoperative screening in an

outpatient clinic setting. ${ }^{1-3}$ Lee $^{4}$ was the first to outline the concept of the preanesthesia evaluation clinic (PAC). He stated that the purpose of the preoperative screening process is "to examine and treat the patient, so that he will arrive in the operating theatre as strong and as healthy as possible", a definition that still adequately defines the process. Today many hospitals operate a PAC. ${ }^{3}$ An accurately performed screening reduces the risk of cancellation on the day of surgery due to the physical condition of the patient, increases the rate of same-day admissions and reduces peri-operative morbidity, resulting in decreased costs and increased quality of care. ${ }^{5,6}$

Congestion is a common phenomenon in outpatient clinics. ${ }^{7-9}$ Patients arriving for a preoperative screening are usually not categorized and therefore the consultation time needed per patient is difficult to estimate. This increases the complexity of the PAC organization as compared with a regular outpatient clinic. In our own PAC, patient waiting times and length of stay were initially significantly shorter than in a comparable clinic $^{10}$, but these increased dramatically after introduction of an electronic patient data management system, since together with the information system additional administrative activities were introduced. Also, the workload of the staff increased, leading to multiple complaints about work stress. The prolonged waiting times, together with the low level of job satisfaction for clinic employees, called for an evaluation of alternative clinic designs. The aim of this study was to explore possibilities for a more efficient operation of our PAC organization. Since all patient movement within the PAC was logged, we chose to use mathematical techniques to analyze performance.

The major advantage of mathematical modeling is the possibility to execute a thorough analysis of a system, while having no impact on the system itself. Using our mathematical model, we 
investigated the effect of various designs on selected performance measures, such as patient length of stay (the total duration of one clinic visit) and staff utilization rate (the fraction of time clinic staff is occupied with patient related activities). One of the alternative designs we considered was regarded as superior to the initial design by the clinic staff. This design was implemented at our PAC in 2007.

The present study was based on a queuing modeling approach. Simulation is a more common approach in this area. Already in 1952, Bailey used Monte-Carlo Simulation to analyze appointment systems for outpatient clinics. ${ }^{11}$ Since then, simulation has been used extensively for the study of outpatient clinics. Within the scope of the PAC, simulation was used to analyze the capacity needed to shorten the waiting list $^{10}$ and to study the design of appointment systems for the PAC to minimize patient waiting times. ${ }^{7}$ The choice for using simulation techniques is not always supported by clear argumentation. ${ }^{12}$ Simulation modeling is a powerful tool, but it is very time-consuming due to the extensive amount of data that is needed and the effort it takes to build the model into a simulation software program. Moreover, it requires detailed information on the input distribution such as those of the consultation time, or the patient arrival processes. Applications of queuing theory in outpatient clinic settings are scarce. The majority of papers published on this matter are covered by Preater in his extensive bibliography on queues in health. ${ }^{13}$ An analytical (queuing) model requires less data and can usually be built in a shorter period of time. ${ }^{14}$ In particular, our queuing modeling approach requires only mean and variance of consultation times and patient arrival processes, and provides robust insight in the underlying relationships of a process, instead of focusing on details as is the case with simulation modeling. As can be seen in Appendix 1, our queuing model consists of several related formulas that can be entered into a spreadsheet. It enables a bottleneck analysis of the processes at the clinic and can easily be adjusted so that it represents one of the alternative designs considered in the redesign process. In this paper we show that queuing theory is a proper alternative for simulation when redesigning processes in an outpatient clinic. Following the intervention, an unexpected increase of $16 \%$ in patient visits in the first quarter of 2008 occurred. However, this did not cause 
a significant increase in waiting times, and in addition resulted in a decrease of employee costs per patient. Furthermore, the time needed to approve a patient for surgery decreased, and employee satisfaction increased. This paper describes the redesign process and provides directions for other PAC managers. 


\section{Methods}

\section{Initial service of the PAC}

The study was performed at a university hospital preanesthesia evaluation clinic, with approximately 6000 patient visits annually. A majority of patients were seen on walk-in basis (about $70 \%$ ), and the remaining on appointment basis. Walk-in patients arrived directly from surgical outpatient clinics within the hospital. Only ASA I or II patients were evaluated on walk-in basis, since for ASA III or IV patients more time for patient contact and additional information from other specialists was often required. It was clinic policy to maximize the number of walk-in patients, although at the same time these patients posed an uncertain demand on clinic resources. Although less than $10 \%$ of patients were classified ASA III or IV and therefore required an appointment, $30 \%$ of all patients were given an appointment. When walk-in patients were deferred to an appointment, it was usually because of overcrowding in the waiting room.

\section{Resources and tasks}

The clinic was run by the department of Anesthesiology, with four anesthesia care providers attending: one staff anesthesiologist, two residents, and a nurse practitioner, supported by a secretary and two clinic assistants. The screening process consisted of at most three steps: an intake at the secretary and two separate contacts with the nurse and anesthesia care provider respectively. All patients would see the secretary and anesthesia care provider, only adults were seen by the clinic assistant. Patients returned to the waiting room between visiting each care provider. Based on a form completed by the referring specialist, the secretary decided whether the patient could be assessed immediately or during an appointment at a later time. Since the secretary is not equipped to make decisions regarding the medical status of the patient, this procedure resulted occasionally in patients receiving an appointment they did not need and vice versa. If the patient received an appointment, the time interval, usually one or two weeks, was used for back-office activities to complete the patient's file. Walk-in patients were approved for surgery by the anesthesia care provider during their visit. The staff anesthesiologist performed 
the back-office activities, consisting mostly of processing additional patient information that was required to finish the case of appointment patients. Because the staff anesthesiologists also served as backup manpower for the front-office activities, they experienced significant work stress. Furthermore, the anesthesia care providers were unhappy because complicated cases had to be finalized by an anesthesia care provider who had not seen the patient initially, which ultimately may result in an incomplete understanding of the medical condition of the patient. ${ }^{15}$

\section{Using Queuing Theory to Analyze PAC Performance}

The initial and alternative designs were compared with a Multi-Class Open Queuing Network Model (for a detailed description see Appendix 1). An advantage of this queuing model is that only the first two moments (mean and standard deviation) of the arrival and service time distributions are needed in the calculations. This allows usage of all possible types of distributions, including empirical distributions. For the comparison two performance measures were calculated with the queuing model, namely patient length of stay and employee utilization rate. In the recent work by Jiang and Giachetti ${ }^{16}$, the authors briefly describe a survey held at their outpatient clinic. From the survey it followed that patients considered the waiting time, being an important contributor to the length of stay, as very important and unsatisfactory long. Other aspects, such as the consultation with the anesthesia care provider and the nurse, also contribute to the patient's contentment on the clinic visit. ${ }^{17}$ Utilization rate and waiting time are related. As an example, consider the single server queue with Poisson arrivals and general service times. The relationship between utilization rate and waiting time is described by the Pollaczek-Khintchine Formula $^{18}$ :

$$
\frac{E(W)}{E(S)}=\frac{\rho}{1-\rho} \frac{1+c^{2}}{2},
$$

where $E(W)$ denotes the expected waiting time, $E(S)$ the expected service time, $\rho$ the utilization rate and $c$ the coefficient of variation of the service time. This non-linear relationship is shown in Figure 1. The utilization rate $\rho$ equals the arrival rate divided by the service rate. The system should be able to deal on average with the offered load $\rho$, and therefore it is required that the 
arrival rate is strictly smaller than the service rate $($ so $\rho<1)$. It is apparent that an increase in the utilization rate from 0.4 to 0.5 has only little impact, whereas an increase from 0.8 to 0.9 results in more than double the original waiting time. An increase from 0.9 to 0.99 even results in an increase of the waiting time by a factor ten. Knowledge of the utilization rate of a system is essential, since increasing this factor when it is already close to one, either by increasing the arrival rate or decreasing the service rate, will result in a considerable increase of the waiting time.

\section{Intervention}

The necessity of an intervention was felt by all parties involved in the PAC. A working group was formed with representatives of all PAC employees. The working group discussed the initial design, and developed four alternative designs, which are described in the subsequent paragraphs. When discussing the initial design, the working group identified all relevant activities at the PAC and standardized the order of these activities in the initial design in several flow charts. Ultimately the working group decided upon the planned design from the presented alternatives. Again, the order of all activities in the new design was documented in several flow charts and medical protocols. The queuing model results were used to support the decision making process and enabled a numerical comparison of the initial and alternative clinic designs.

\section{Alternative design 1: Clinic Assistant Selects at Front Desk}

Clinic assistants were convinced that many patients with an actual ASA III or IV score were assigned an erroneous ASA I or II score by the secretary. These patients were treated on walk-in basis and consumed too much time in the office of the anesthesia care provider, resulting in congestion in the waiting room. They suggested that one of the clinic assistants should take over part of the front desk task from the secretary, while the other clinic assistant performs measurements and blood sampling.

\section{Alternative design 2: Treat all Patients on Appointment Basis}


Demand for an outpatient clinic's services can be divided into two components: controlled (appointment patients) and uncontrolled (walk-in patients) demand..$^{19}$ In the initial set-up most ASA I or II patients were seen as walk-in patients. In the second alternative all patients are deferred to an appointment, since a clinic with an appointment-only system will always provide a better service level than a clinic that allows walk-in arrivals. ${ }^{7}$

\section{Alternative design 3: Reschedule Appointments}

Rising ${ }^{19}$ suggested to schedule appointments such that they complement walk-in arrivals. This results in a more homogeneous arrival pattern throughout the day. In the PAC under study the number of walk-in arrivals was significantly lower in the early morning and on Friday afternoon. In this alternative all appointments are scheduled in these periods.

\section{Alternative design 4: Regroup Employee Tasks and Amend Patient Flows}

In this alternative the secretary accepts all patients; therefore all patients are seen by the clinic assistant on their first visit. Clinic assistants are provided with protocols to aid in the decision whether the patient can be seen immediately based on the extent of co-morbidity, contacts with medical specialists and the requirement to obtain additional medical information prior to the visit to the anesthesiologist. If the patient requires additional testing, these tests are immediately performed and/or requested and the patient is deferred to an appointment, scheduled when all additional information is available. Consequently, the patient can be approved for surgery when the appointment takes place. 


\section{Results}

\section{Model input}

Data from all PAC visits recorded in the first quarter of 2007 was used to obtain input parameters for the queuing model $(n=1492)$. For the analysis, patients were divided in three separate classes: (1) children (<16 years old), (2) adult patients ASA Score I or II, and (3) adult patients ASA Score III or IV. This classification was chosen since children and adults have a different routing, moreover the three classes can be distinguished in consultation time at the anesthesiologist. An advantage of this classification is that it is similar to that used by clinic staff. Arrival rates for each patient class, and mean and standard deviation of the contact time at the clinic assistant and anesthesia care provider were determined. Not all registered contacts were stored correctly and therefore the records of 1293 patients (87\%) could be used for the latter part of the data analysis. The time patients spent at the secretary was not recorded and therefore estimated. The secretary was often disturbed by co-workers who inquire after the approval status of a particular patient, either by phone or in person at the reception desk. The anesthesia care providers were also disturbed by co-workers, the inquiries usually concerning their other professional responsibilities. We estimated that $50 \%$ of the secretary's time, and $33 \%$ of the anesthesia care provider's time, was lost due to these inevitable interferences. The number of arrivals per patient class was used to determine the distribution of patients among classes. We found that the majority of patients arrived between 10 AM and 4 PM. Hence we focused our analysis on this interval and calculated the arrival rate ( 3.73 patients/hour) by using patient arrivals recorded during this interval. We observed that within this period, patients from all classes arrived in a homogenously distributed manner. This corresponds with the squared coefficient of variation (see Appendix 1) of the arrival process being equal to 1 for all patient classes. The arrivals of patients that were immediately deferred to an appointment were not recorded. Assuming that all appointment patients make their appointment at the reception desk, we calculated the arrival rate of non-admitted patients by multiplying the admitted patient arrival rate by the appointment percentage for each patient class. A summary of input data is given in Table 
1. Senior clinic staff members discussed and carefully checked all parameter values; additionally they discussed and approved the queuing model design.

\section{Comparison of Initial Design and Alternatives}

With the model, we compared each alternative design with the initial design. If necessary, input parameters were adjusted (see Table 2 for the modifications and Appendix 1 for an explanation of the parameters itself). The performance measures we chose to compare were expected patient length of stay and employee utilization rate. The initial design could be characterized by long expected patient length of stays, caused by prolonged waiting times at the secretary and later in the process, prior to the contact with the anesthesia care provider (Table 3). These two care stations also had high utilization rates. Comparing the performance measures of the initial design to those of the alternative designs lead to the conclusion that all alternative designs, except alternative 2 (treat all patients on appointment basis) would result in a better overall performance.

Once the model results were available, the working group was consulted to make a decision on the next step to take in the redesigning process. It was apparent to all members that the initial design could not be maintained. The first alternative of relocating one nurse to the secretary's station was not regarded as a valuable alternative, since the expected decrease in patient length of stay was minimal. Furthermore, patient waiting time at the remaining clinic assistant increased substantially, which was also undesirable. Based on the predicted increase in patient waiting time at the secretary in alternative 2 , which was caused by all patients having to make an appointment first, and since introducing an appointment-only system was regarded as patient unfriendly (in the sense of one-stop shopping) by the working group, alternative 2 was eliminated. The working group members decided to implement alternative 3 and 4, so that advantages of both alternatives were included. The effects of combining alternatives 3 and 4 were again studied with the queuing model (Table 3). The queuing model predicted that this intervention would also result in an improvement. Supported by the results, all working group members were convinced that 
implementing a combination of the two alternatives would yield a better overall performance of the clinic.

\section{Effect of intervention}

The new design was implemented in the summer of 2007. We compared total patient length of stay before and after the intervention. To minimize seasonal influences and to allow for learning effects, we used data from both the first quarter of 2007 and 2008. Before the intervention, only one clinic assistant was present on Fridays. Since the intervention involved scheduling the majority of appointments on Fridays, one additional clinic assistant shift was now required. This caused an increase in total employee capacity from 7.20 FTE (total costs: $109 \mathrm{~K}$ Euros) to 7.87 FTE (total costs: $116 \mathrm{~K}$ Euros, $+6 \%$ ). Before the intervention, total patient length of stay was on average 70.57 minutes (95\% Cl: [68.76; 72.38]), compared to 77.91 minutes after the intervention (95\% Cl: $[76.14 ; 79.68])$. The increase in patient length of stay was caused solely by longer contact and waiting times at the clinic assistant $(95 \% \mathrm{Cl}$ of increase in contact times: [5.52; 7.60], $95 \% \mathrm{Cl}$ of increase in waiting times: $[7.96 ; 11.94])$. The contact and waiting times at the anesthesia care provider did not increase significantly $(95 \% \mathrm{Cl}$ of increase in contact times: [$1.50 ; 1.46], 95 \% \mathrm{Cl}$ of increase in waiting times: $[-1.02 ; 2.38])$. Recall that not all patients see the nurse and therefore the increase in total patient length of stay was less. In the first quarter of 2008, 1737 patient contacts were registered during the opening hours of the clinic, an increase of 245 patients $(+16 \%)$ compared to the first quarter of 2007 . Dividing the total personnel costs by the number of patients for both quarters, we see that personnel costs decreased from 73 to 67 Euros per patient (-8\%). The percentage of patients seen on walk-in basis increased from $72 \%$ in 2007 to $81 \%$ in 2008 . This is considered an improvement in patient friendliness. Furthermore, in 2008 the anesthesiologist needed 6.8 days to decide upon approving the patient for surgery, compared to 7.9 days in 2007 (95\% Cl: [-0.3; 2.3]).. The staff anesthesiologists were responsible for finalizing the status of those patients for which new information was obtained in the days or weeks after the patient had visited the PAC. This resulted in a considerable expense of time (several hours), since they had to make themselves fully acquainted with the patient's file before 
they could approve the patient. After the intervention this task was minimal (less than 30 minutes), as for most patients all relevant information was available prior to the first visit to the attending anesthesia care provider. 


\section{Discussion}

In this paper we have discussed the process of redesigning our PAC, a facility with both walk-in and appointment patients. We demonstrated a queuing modeling approach that enables a fast and robust analysis of PAC performance. The methodology can be applied to other preoperative screening clinics as well. The redesign consisted of two parts, namely the re-scheduling of appointments to the early morning and Friday, and the reassignment of tasks from the secretary to the clinic assistants. As a consequence, all patients were seen on their walk-in visit by the clinic assistant. Patients requiring more contact time with the anesthesia care provider or backoffice activities were deferred to an appointment by the clinic assistant, scheduled when all required information was available. Literature about the re-design of hospital care is extensive. ${ }^{20}$ However, the literature on re-design of outpatient and preanesthesia evaluation clinics is limited. Some studies are dedicated to the design of appointment systems ${ }^{7}$, others concentrated largely on waiting times and patient satisfaction..$^{10,21}$ The concept of re-design by reallocating tasks at the outpatient clinic has received less attention.

A possible limitation of this study is that all outcomes of the queuing model were calculated under the assumption of steady state behavior. The system under study will never reach this equilibrium state, due to inhomogeneous patient arrivals and restrictive opening hours. Under similar restrictions, queuing theory has been successfully applied in other areas such as manufacturing. ${ }^{22}$ Therefore we feel that this approach maintains its suitability. Furthermore, we used the queuing model solely for comparison purposes and not for prediction of actual patient length of stay and utilization rates, which further strengthened our belief in the followed approach.

The model enabled us to analyze the effect of increased pressure on the clinic. As mentioned in the results section, patient arrivals had increased with $16 \%$ in the first quarter of 2008 , compared to the same period in 2007. Nevertheless, empirical analysis showed that patient length of stay had only increased slightly. The model shows that the rise in patient arrivals would have resulted 
in a tremendous increase in patient length of stay and employee utilization rate, if we had not changed the design of our PAC (Table 4). Under the 2008 data the initial design operates under high pressure, with an increase in length of stay of $53 \%$, due to the $16 \%$ increase in patient arrivals. In the implemented design, due to increased efficiency, the system operates under modest pressure, with an increase in length of stay of only $15 \%$ (Table 4). This is in line with the relationship depicted in Figure 1, indicating the typical relation between waiting time and load. By organizing the processes at the clinic more efficiently, we reduced the load and moved left on the curve. Therefore, the increase in patient arrivals did cause an increase in the load but only a slight increase in waiting time, and patient length of stay.

The majority of patients visiting our PAC are seen on a walk-in basis. Since patients have the opportunity to go straight from the surgical outpatient clinic to the PAC, they are often able to finalize the entire preoperative preparation within one hospital visit (one-stop shopping), avoiding multiple hospital visits. However, walk-in outpatient clinics are notoriously more difficult to handle in terms of optimizing waiting times for patients and peak pressures for anesthesia care providers. Dexter ${ }^{7}$ states that the best service walk-in PACs can provide will always be worse than appointment PACs. The walk-in PAC requires more resources to have acceptable waiting times for patients ${ }^{10}$, since more slack is required to deal with unexpected peaks in patient arrival. Appointment systems on the other hand deal with peaks in demand for PAC services by building waiting lists. To allow for patients that need to be seen with some urgency, these appointmentonly outpatient clinics will usually have some unplanned time slots (or add-on manpower). At the PAC under study, we use a system that allows both walk-in and appointment patients. The decrease of back-office activities enabled the anesthesia care providers to dedicate more time to patient contact. This explains how $16 \%$ more patients could be seen without an increase in the number of anesthesia care providers.

Although the model aided in the re-design of the PAC, the success of the intervention relied heavily on the involvement of the employees. The key to a successful intervention is the 
combination of logistic expertise and the participation of key persons in the project. ${ }^{23}$ Therefore the outcomes of the queuing model regarding the performance of the initial and alternative designs were discussed in a working group with representatives of all clinic employees. We feel that the intervention's success was in large part the result of a committed working group, together with the use of mathematical modeling tools enabling quantified comparisons of alternative clinic designs. 


\section{Figures}

\section{Figure 1}

Title: The Pollaczek-Khintchine Curve ${ }^{a}$

Figure 2

Title: Single Class Open Queuing Network

${ }^{a} E(W)=$ Mean waiting time, $E(S)=$ Mean service time 


\section{Appendix 1: The Queuing Model}

With the multi-class open queuing network model, we derived measures to analyze performance of our PAC. There are many books that give an introduction to Queuing Theory, see for example Taylor and Karlin. ${ }^{24}$ The extensive bibliography by Preater ${ }^{13}$ provides many examples of Queuing Theory applications in health care. The paper by Jiang and Giachetti ${ }^{16}$ gives an example of a multi-class open queuing network model applied to an outpatient clinic. A queuing network model represents a set of connected queues. At the PAC there are three separate queues where the employees act as servers. The secretary station is a single server queue whereas the clinic assistant and anesthesia care provider station are multi-server queues. Patients enter the queuing network via the secretary station and finally depart from the system. Furthermore, if upon arrival at a station an employee is available patients are served immediately; otherwise they join the queue in first come first serve discipline. We use the approximate decomposition method ${ }^{25}$ to analyze the model. The approximate decomposition method has as advantage that only the mean and squared coefficient of variation (SCV) of the arrival process and contact times are needed (the SCV equals the variance divided by the squared mean).

First we introduce some notations. There are $r$ distinct patient classes, where $r=1$ are patients deferred to an appointment by the secretary, $r=2$ adults ASA I or II, $r=3$ adults ASA III or IV, and $r=4$ are children. To evaluate alternative design 4 we also introduce $r=5,6,7$ to represent patients (adults with ASA I or II, adults with ASA III or IV, and children respectively) who return for their appointment. These indexes only exist when alternative design 4 is evaluated, and therefore the binary variable $d$ equals 1 if alternative design 4 is evaluated and 0 otherwise. We have $i$ stations, $i=[1 . .3]$ representing the secretary, clinic assistant and anesthesia care provider respectively. The number of servers (i.e. employees) at station i equals $\mathrm{s}_{\mathrm{i}}$. An effective capacity of $e_{1}$ for the secretary, and $e_{3}$ for the anesthesia care is introduced. Since the secretary is disturbed while handling patients, $e_{1}$ is taken into account when calculating the mean time a patient spends 
at this station. The anesthesia care provider is not disturbed while treating patients and therefore $e_{3}$ is only used in calculating the utilization rate. The arrival rate of patient class $r$ at station 1 is $\zeta_{r}$, whereas $E\left(S_{r, i}\right)$ is the mean service time for patient class $r$ at station i. The SCV of arrivals at station 1 of patient class $r$ is $\operatorname{SCV}_{A, r, 1}$ and $S C V_{S, r, i}$ is the SCV of the service time for patient class $r$ at station i. Finally, $a_{r}$ is the fraction of patients of class $r$ who are deferred to an appointment in alternative design 4 . In the model analysis we follow the three steps from the approximate decomposition method.

\section{Step 1: Reduction to a Single Class Open Queuing Network}

Figure 2 gives the Single Class OQN resulting from the aggregation of the patient flows. The aggregated arrival intensities at station i

$\lambda_{1}=\sum_{r=1+d}^{4+3 d} \varsigma_{r}, \quad \lambda_{2}=\sum_{r=2}^{3} \varsigma_{r}, \quad \lambda_{3}=\sum_{r=2}^{4}\left(1-d a_{r}\right) \varsigma_{r}+d \sum_{r=5}^{7} \varsigma_{r}$

The utilization rates per patient class for station 1,2,and 3 are

$\rho_{1, r}=\varsigma_{r} E\left(S_{r, 1}\right) \frac{1}{e_{1} s_{1}}$ for $r=1+d, \ldots, 4+3 d, \quad \rho_{2, r}=\varsigma_{r} E\left(S_{r, 2}\right) \frac{1}{s_{2}} \quad$ for $r=2,3$, and

$\rho_{3, r}=\varsigma_{r} E\left(S_{r, 3}\right) \frac{1}{e_{3} s_{3}}+d\left(1-a_{r}\right) \varsigma_{r} E\left(S_{r, 3}\right) \frac{1}{e_{3} s_{3}}$ for $r=2, \ldots, 4+3 d$.

Aggregating the utilization rates over all patient classes for station 1,2,3 gives

$\rho_{1}=\sum_{r=1+d}^{4+3 d} \rho_{1, r}, \quad \rho_{2}=\sum_{r=2}^{3} \rho_{2, r}, \quad \rho_{3}=\sum_{r=2}^{4+3 d} \rho_{3, r}$

For stability it is required that $\rho_{\mathrm{i}}<1$ for all $\mathrm{i}$.

\section{Step 2: Analysis of the Single Class Open Queuing Network}

The arrival process at station 1 has SCV

$S C V_{A, 1}=w_{1} \sum_{r=1+d}^{4+3 d} Q_{r, 1} S C V_{A, r, 1}+1-w_{1}$, where
$w_{1}=\frac{1}{1+4\left(1-\rho_{1}\right)^{2}\left(v_{1}-1\right)}, \quad v_{1}=\frac{\lambda_{1}^{2}}{\sum_{r=1+d}^{4+3 d} \varsigma_{r}^{2}}, \quad Q_{r, 1}=\frac{\varsigma_{r}}{\lambda_{1}}$.

The mean contact time and SCV at station 1 are 
$E\left(S_{1}\right)=\frac{1}{\lambda_{1}} \sum_{r=1+d}^{4+3 d} \varsigma_{r} E\left(S_{r, 1}\right), \quad S C V_{S, 1}=\frac{1}{\lambda_{1} E^{2}\left(S_{1}\right)} \sum_{r=1+d}^{4+3 d} \varsigma_{r} E^{2}\left(S_{r, 1}\right)\left(S C V_{S, r, 1}+1\right)-1$

The arrival process at station 2 has SCV

$S C V_{A, 2}=P_{1,2} S C V_{D, 1}+1-P_{1,2}$

where $S C V_{D, 1}$ is the SCV of the departure process at station 1 and $P_{1,2}$ is the portion of the aggregated flow out of station 1 to station 2

$S C V_{D, 1}=\left(1-\rho_{1}^{2}\right) S C V_{A, 1}+\rho_{1}^{2} S C V_{S, 1}, \quad P_{1,2}=\frac{\sum_{r=2}^{3} \varsigma_{r}}{\lambda_{1}}$

Station 2 has mean contact time and SCV

$E\left(S_{2}\right)=\frac{1}{\lambda_{2}} \sum_{r=2}^{3} \varsigma_{r} E\left(S_{r, 2}\right), \quad S C V_{S, 2}=\frac{1}{\lambda_{2} E^{2}\left(S_{2}\right)} \sum_{r=2}^{3} \varsigma_{r} E^{2}\left(S_{r, 2}\right)\left(S C V_{S, r, 2}+1\right)-1$

The arrival process at station 3 has SCV

$S C V_{A, 3}=w_{3}\left(Q_{2,3} S C V_{2,3}+Q_{1,3} S C V_{1,3}\right)+1-w_{3}$, with

$w_{3}=\frac{1}{1+4\left(1-\rho_{3}\right)^{2}\left(v_{3}-1\right)}, \quad v_{3}=\frac{1}{Q_{2,3}^{2}+Q_{1,3}^{2}}, \quad Q_{2,3}=\sum_{r=2}^{3} \frac{\left(1-d a_{r}\right) \varsigma_{r}}{\lambda_{3}}$,

$Q_{1,3}=\frac{\sum_{r=4}^{4+3 d}\left(1-d a_{r}\right) \varsigma_{4}}{\lambda_{3}}, \quad S C V_{1,3}=P_{1,3} S C V_{D, 1}+1-P_{1,3}, \quad P_{1,3}=\frac{\sum_{r=4}^{4+3 d}\left(1-d a_{r}\right) \varsigma_{4}}{\lambda_{1}}$,

$S C V_{2,3}=(1-d) S C V_{D, 2}+d\left(P_{2,3} S C V_{D, 2}+1-P_{2,3}\right), \quad P_{2,3}=\sum_{r=2}^{3} \frac{\left(1-d a_{r}\right) \varsigma_{r}}{\lambda_{2}}$,

$S C V_{D, 2}=1+\left(1-\rho_{2}^{2}\right)\left(S C V_{A, 2}-1\right)+\frac{\rho_{2}^{2}}{\sqrt{s_{2}}}\left(S C V_{S, 2}-1\right)$

where $\mathrm{SCV}_{2,3}$ is the SCV of the patient flow from station 2 to station $3, \mathrm{SCV}_{1,3}$ the SCV of the patient flow from station 1 to station 3 , and $S_{C V} V_{D, 2}$ is the SCV of the departure process at station 2. $P_{1,3}$ is the portion of aggregated flow out of station 1 to station 3 , and $P_{2,3}$ is the portion of aggregated flow out of station 2 to station 3.

Station 3 has mean contact time and SCV 


$$
\begin{aligned}
& E\left(S_{3}\right)=\frac{1}{\lambda_{3}} \sum_{r=2}^{4}\left(1-d a_{r}\right) \varsigma_{r} E\left(S_{r, 3}\right)+d \sum_{r=5}^{7} \varsigma_{r} E\left(S_{r, 3}\right) \\
& S C V_{S, 3}=\frac{1}{\lambda_{3} E^{2}\left(S_{3}\right)}\left(\sum_{r=2}^{4}\left(1-d a_{r}\right) \varsigma_{r} E^{2}\left(S_{r, 3}\right)\left(S C V_{S, r, 3}+1\right)+\sum_{r=5}^{7} \varsigma_{r} E^{2}\left(S_{r, 3}\right)\left(S C V_{S, r, 3}+1\right)\right)-1
\end{aligned}
$$

\section{Step 3: Performance Measures per Patient Class}

We are interested in the waiting times for patients per station and the utilization rates per

employee at each station. The latter is given by the aggregated utilization rates derived in step 1, while the mean waiting times are obtained by using the SCV's and mean service times calculated in step 2. The mean waiting time at the three service stations is equal for all patient classes.

$$
E\left(W_{Q, 1}\right)=\frac{S C V_{A, 1}+S C V_{S, 1}}{2} \frac{\rho_{1}}{1-\rho_{1}} \frac{E\left(S_{1}\right)}{e_{1}}, \quad E\left(W_{Q, i}\right)=\frac{S C V_{A, i}+S C V_{S, i}}{2} E\left(W_{Q, i(M / M / c)}\right),
$$

where $E\left(W_{Q, i(M / M / c)}\right)=\frac{\left(s_{i} \rho_{i}\right)^{s i}}{s_{i} ! G_{i}} \frac{1}{\left(1-\rho_{i}\right)^{2}} \frac{E\left(S_{i}\right)}{s_{i}}, \quad G_{i}=\sum_{n=0}^{s_{i}-1} \frac{\left(s_{i} \rho_{i}\right)^{n}}{n !}+\frac{\left(s_{i} \rho_{i}\right)^{s_{i}}}{\left(1-\rho_{i}\right) s_{i} !}$ for $\mathrm{i}=2,3$.

Patient length of stay for each patient class can now be calculated by adding the mean waiting and length of stay of all care stations the patient calls at on his visit to the PAC. 


\section{References}

1. Conway JB, Goldberg J, Chung F. Preadmission anaesthesia consultation clinic. Can J Anaesth 1992;39:1051-7

2. Lew E, Pavlin DJ, Amundsen L. Outpatient preanaesthesia evaluation clinics. Singapore Med J 2004;45:509-16

3. Pollard JB. Economic aspects of an anesthesia preoperative evaluation clinic. Curr Opin Anaesthesiol 2002;15:257-61

4. Lee JA. The Anaesthetic Out-Patient Clinic. Anaesthesia 1949;4:169-74

5. van Klei WA, Moons KG, Rutten CL, Schuurhuis A, Knape JT, Kalkman CJ, Grobbee $D E$. The effect of outpatient preoperative evaluation of hospital inpatients on cancellation of surgery and length of hospital stay. Anesth Analg 2002;94:644-9

6. Parker BM, Tetzlaff JE, Litaker DL, Maurer WG. Redefining the preoperative evaluation process and the role of the anesthesiologist. J Clin Anesth 2000;12:350-6

7. Dexter F. Design of appointment systems for preanesthesia evaluation clinics to minimize patient waiting times: a review of computer simulation and patient survey studies. Anesth Analg 1999;89:925-31

8. Dunnill MG, Pounder RE. Medical outpatients: changes that can benefit patients. Clin Med 2004;4:45-9

9. Harper PR, Gamlin HM. Reduced outpatient waiting times with improved appointment scheduling: a simulation modelling approach. Or Spectrum 2003;25:207-22

10. Edward GM, Razzaq S, de Roode A., Boer F, Hollmann MW, Dzoljic M, Lemaire LC. Patient flow in the preoperative assessment clinic. Eur J Anaesthesiol 2008;25:280-6

11. Bailey NTJ. A study of Queues and Appointment Systems in Hospital Out-Patient Departments, with Special Reference to Waiting-Times. Journal of the Royal Statistical Society Series B-Statistical Methodology 1952;14:185-99

12. de Bruin AM, van Rossum AC, Visser MC, Koole GM. Modeling the emergency cardiac in-patient flow: an application of queuing theory. Health Care Manag Sci 2007;10:125-37

13. Preater J. Queues in health. Health Care Manag Sci 2002;5:283

14. Green L. Queueing Analysis in Healthcare. In: Hall RW, ed. Patient Flow: Reducing Delay in Healthcare Delivery. 1 ed. New York, NY: Springer, 2006:281-307

15. Gibby GL, Schwab WK. Availability of records in an outpatient preanesthetic evaluation clinic. J Clin Monit Comput 1998;14:385-91

16. Jiang L, Giachetti RE. A queueing network model to analyze the impact of parallelization of care on patient cycle time. Health Care Manag Sci 2008;11:248-61

17. Edward GM, de Haes JC, Oort FJ, Lemaire LC, Hollmann MW, Preckel B. Setting priorities for improving the preoperative assessment clinic: the patients' and the professionals' perspective. Br J Anaesth 2008;100:322-6 
18. Cohen JW. The Single Server Queue. 8 ed. Amsterdam, the Netherlands: NorthHolland Publishing Company, 1982

19. Rising EJ, Baron R, Averill B. A Systems Analysis of a University-Health-Service Outpatient Clinic. Operations Research 1973;21:1030-47

20. Elkhuizen SG, Limburg M, Bakker PJ, Klazinga NS. Evidence-based re-engineering: re-engineering the evidence--a systematic review of the literature on business process redesign (BPR) in hospital care. Int J Health Care Qual Assur Inc Leadersh Health Serv 2006;19:477-99

21. Hepner DL, Bader AM, Hurwitz S, Gustafson M, Tsen LC. Patient satisfaction with preoperative assessment in a preoperative assessment testing clinic. Anesth Analg 2004;98:1099-105

22. Buzacott JA, Shanthikumar JG. Stochastic Models of Manufacturing Systems. 1 ed. Englewood Cliffs, NJ: Prentice Hall, 1993

23. Elkhuizen SG, Burger MP, Jonkers RE, Limburg M, Klazinga N, Bakker PJ. Using business process redesign to reduce wait times at a university hospital in the Netherlands. Jt Comm J Qual Patient Saf 2007;33:332-41

24. Taylor HM, Karlin S. An Introduction to Stochastic Modeling. 3 ed. San Diego, CA: Academic Press, 1998

25. Bitran GR, Morabito R. Survey Open Queueing Networks: Optimization and Performance Evaluation Models for Discrete Manufacturing Systems. Production and Operations Management 1996;5:163-93 
Table 1

Title: Summary of Input Data

\begin{tabular}{|c|c|c|c|c|c|c|c|c|c|}
\hline \multirow[t]{2}{*}{ Patient class } & \multirow[t]{2}{*}{$\mathbf{N}$} & \multirow[t]{2}{*}{$\begin{array}{l}\text { Appointment } \\
\text { Percentage }\end{array}$} & \multirow[t]{2}{*}{$\begin{array}{l}\text { Arrival } \\
\text { rate }\end{array}$} & \multicolumn{2}{|c|}{$\begin{array}{l}\text { Service Time } \\
\text { Secretary }\end{array}$} & \multicolumn{2}{|c|}{$\begin{array}{l}\text { Service Time } \\
\text { Clinic Assistant }\end{array}$} & \multicolumn{2}{|c|}{$\begin{array}{l}\text { Service Time } \\
\text { Anesthesia Care Provider }\end{array}$} \\
\hline & & & & $E(\mathbf{S})^{b}$ & $\mathbf{S D}(\mathbf{S})^{c}$ & $E(S)$ & SD(S) & $E(S)$ & SD(S) \\
\hline Children & 274 & $15 \%$ & 0.79 & 5.00 & 5.00 & - & - & $\begin{array}{c}24.30 \\
(n=274)\end{array}$ & 20.64 \\
\hline $\begin{array}{l}\text { Adults ASA } \\
\text { I or II }\end{array}$ & 902 & $25 \%$ & 2.60 & 5.00 & 5.00 & $\begin{array}{c}10.71 \\
(n=711)\end{array}$ & 8.97 & $\begin{array}{c}27.24 \\
(n=902)\end{array}$ & 17.26 \\
\hline $\begin{array}{l}\text { Adults ASA } \\
\text { III or IV }\end{array}$ & 117 & $78 \%$ & 0.34 & 5.00 & 5.00 & $\begin{array}{l}16.31 \\
(n=86)\end{array}$ & 14.20 & $\begin{array}{c}52.05 \\
(n=117)\end{array}$ & 25.50 \\
\hline $\begin{array}{l}\text { Deferred to } \\
\text { appointment }\end{array}$ & - & - & 1.04 & 2.50 & 2.50 & - & - & - & - \\
\hline
\end{tabular}

${ }^{b}$ Mean service time in minutes

${ }^{c}$ Standard deviation of service time in minutes 
Table 2: Adjustment of Input Parameters for Evaluation of Alternative Designs ${ }^{d}$

\begin{tabular}{|c|c|c|}
\hline Alternative & Adjustment & Explanation \\
\hline $1^{e}$ & $\begin{array}{l}\text { - } s_{2}=1 \\
\text { - } e_{1}=1\end{array}$ & $\begin{array}{l}\text { One clinic assistant moves to secretary station } \\
\text { No disturbance during welcoming of patients }\end{array}$ \\
\hline $2^{f}$ & $\begin{array}{l}\text { - } \zeta_{1}=3.73 \\
\text { - } \operatorname{SCV}_{\mathrm{A}, 2,1}=0 \\
\text { - } \operatorname{SCV}_{\mathrm{A}, 3,1}=0 \\
\operatorname{SCV}_{\mathrm{A}, 4,1}=0\end{array}$ & $\begin{array}{l}\text { The secretary gives all patients an appointment the first } \\
\text { time they arrive at the PAC, thus arrival rate increases. } \\
\text { We assume that appointment patients arrive on time. } \\
\text { Therefore the standard deviation of their arrival time } \\
\text { equals } 0 \text {, which results in an SCV equal to } 0 .\end{array}$ \\
\hline $3^{g}$ & $\begin{array}{l}\text { - } \zeta_{2}=1.93 \\
\text { - } \zeta_{3}=0.07 \\
\zeta_{4}=0.67\end{array}$ & $\begin{array}{l}\text { Appointments are rescheduled outside the interval } \\
10 \mathrm{AM}-4 \mathrm{PM} \text { and therefore the fraction of patients with } \\
\text { an appointment is removed from the arrival rates. }\end{array}$ \\
\hline $4^{h}$ & $\begin{array}{ll}\text { - } & \zeta_{1}=0 \\
\text { - } & \mathrm{E}\left(\mathrm{S}_{\mathrm{r}, 1}\right)=2.50 \\
\text { - } & \mathrm{E}\left(\mathrm{S}_{2,2}\right)=15.71^{\mathrm{i}} \\
\text { - } & \mathrm{E}\left(\mathrm{S}_{3,2}\right)=21.31 \\
& \\
\text { - } & \mathrm{SCV}_{\mathrm{A}, 5,1}=0 \\
& \mathrm{SCV}_{\mathrm{A}, 6,1}=0 \\
& \mathrm{SCV}_{\mathrm{A}, 7,1}=0\end{array}$ & $\begin{array}{l}\text { No patients are deferred to an appointment by the } \\
\text { secretary. } \\
\text { Consultation time at secretary decreases with } 2.5 \\
\text { minutes, since part of tasks are reallocated to clinic } \\
\text { assistants; consultation times at clinic assistants } \\
\text { increases with these } 2.5 \text { minutes and with an additional } \\
2.5 \text { minutes needed to determine upon additional } \\
\text { testing. } \\
\text { We assume that appointment patients arrive on time. } \\
\text { Therefore the standard deviation of their arrival time } \\
\text { equals } 0 \text {, which results in an SCV equal to } 0 .\end{array}$ \\
\hline
\end{tabular}

\footnotetext{
${ }^{\mathrm{d}}$ All other parameter values remain constant

${ }^{e}$ Clinic assistant selects at front desk

${ }^{f}$ Treat all patients on appointment basis

${ }^{g}$ Reschedule appointments

${ }^{h}$ Regroup employee tasks and amend patient flows
} 
${ }^{i}$ We assumed that the ratio between expectation and variance of the contact time at the clinical assistants (and therefore also the SCV) remained constant. 
Table 3

Title: Results of Analytical Model

\begin{tabular}{|c|c|c|c|c|c|c|c|}
\hline \multirow[t]{2}{*}{ Design } & \multicolumn{2}{|c|}{ Secretary } & \multicolumn{2}{|c|}{ Clinic Assistant } & \multicolumn{2}{|c|}{ Anesthesia Care Provider } & \multirow[t]{2}{*}{ Patient length of stay } \\
\hline & $\rho^{k}$ & $\mathrm{E}(\mathrm{W})^{\prime}$ & $\rho$ & $E(W)$ & $\rho$ & $E(W)$ & \\
\hline Initial & 0.68 & 19.20 & 0.28 & 0.60 & 0.67 & 9.60 & 77.35 \\
\hline${\text { Alternative } \mathrm{I}^{m}}^{m}$ & 0.34 & 2.40 & 0.56 & 12.60 & 0.67 & 9.00 & 71.95 \\
\hline${\text { Alternative } \text { II }^{n}}$ & 0.90 & 54.00 & 0.28 & 0.60 & 0.67 & 9.60 & 107.15 \\
\hline Alternative III $^{\circ}$ & 0.51 & 9.60 & 0.18 & 0.60 & 0.45 & 1.80 & 59.95 \\
\hline Alternative IV $^{p}$ & 0.38 & 3.00 & 0.40 & 2.40 & 0.67 & 9.60 & 62.95 \\
\hline Alternative III + IV & 0.30 & 2.04 & 0.40 & 2.63 & 0.44 & 1.60 & 54.22 \\
\hline
\end{tabular}

\footnotetext{
${ }^{j}$ Mean patient length of stay in minutes, for the most common group of patients (adult ASA I/II walk-in)

${ }^{k}$ Occupation rate

Mean waiting time in minutes

${ }^{m}$ Clinic assistant selects at front desk

${ }^{n}$ Treat all patients on appointment basis

${ }^{\circ}$ Reschedule appointments

${ }^{p}$ Regroup employee tasks and amend patient flows
} 
Table 4

Title: Results of Analytical Model with 2008 Data $^{q}$

\begin{tabular}{|c|c|c|c|c|c|c|c|}
\hline Design & Secr & tary & Clini & Assistant & Anes & esia Care Provider & Patient length of stay ${ }^{r}$ \\
\hline & $\rho^{s}$ & $\mathrm{E}(\mathrm{W})^{t}$ & $\rho$ & $E(W)$ & $\rho$ & $E(W)$ & \\
\hline Initial & 0.81 & 38.24 & 0.35 & 1.46 & 0.83 & 30.38 & 118.03 \\
\hline Alternative III + IV ${ }^{u}$ & 0.37 & 2.80 & 0.50 & 4.75 & 0.63 & 6.70 & 62.20 \\
\hline
\end{tabular}

${ }^{9}$ Arrival rates: Children 0.87, Adults ASA I or II 3.32, Adults III or IV 0.41 , deferred to appointment 0.88

${ }^{r}$ Mean patient length of stay in minutes, for the most common group of patients (adult ASA I/II walk-in)

${ }^{s}$ Occupation rate

${ }^{t}$ Mean waiting time in minutes

${ }^{u}$ Reschedule appointments, regroup employee tasks and amend patient flows 


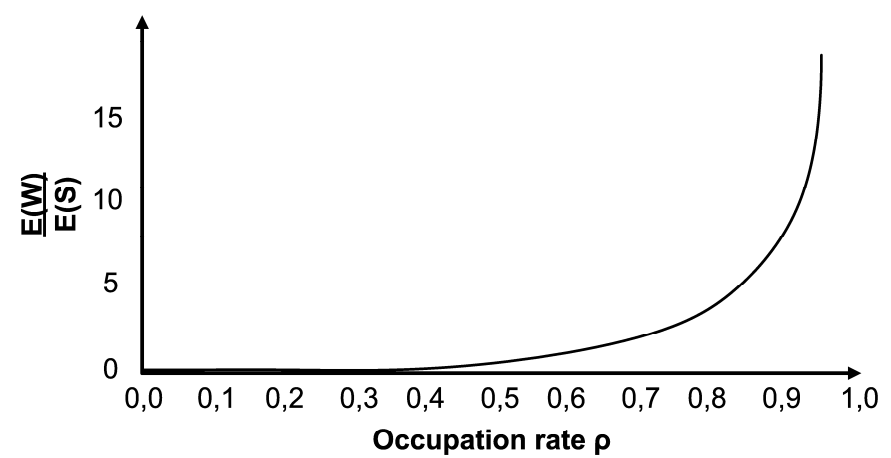

Figure 1

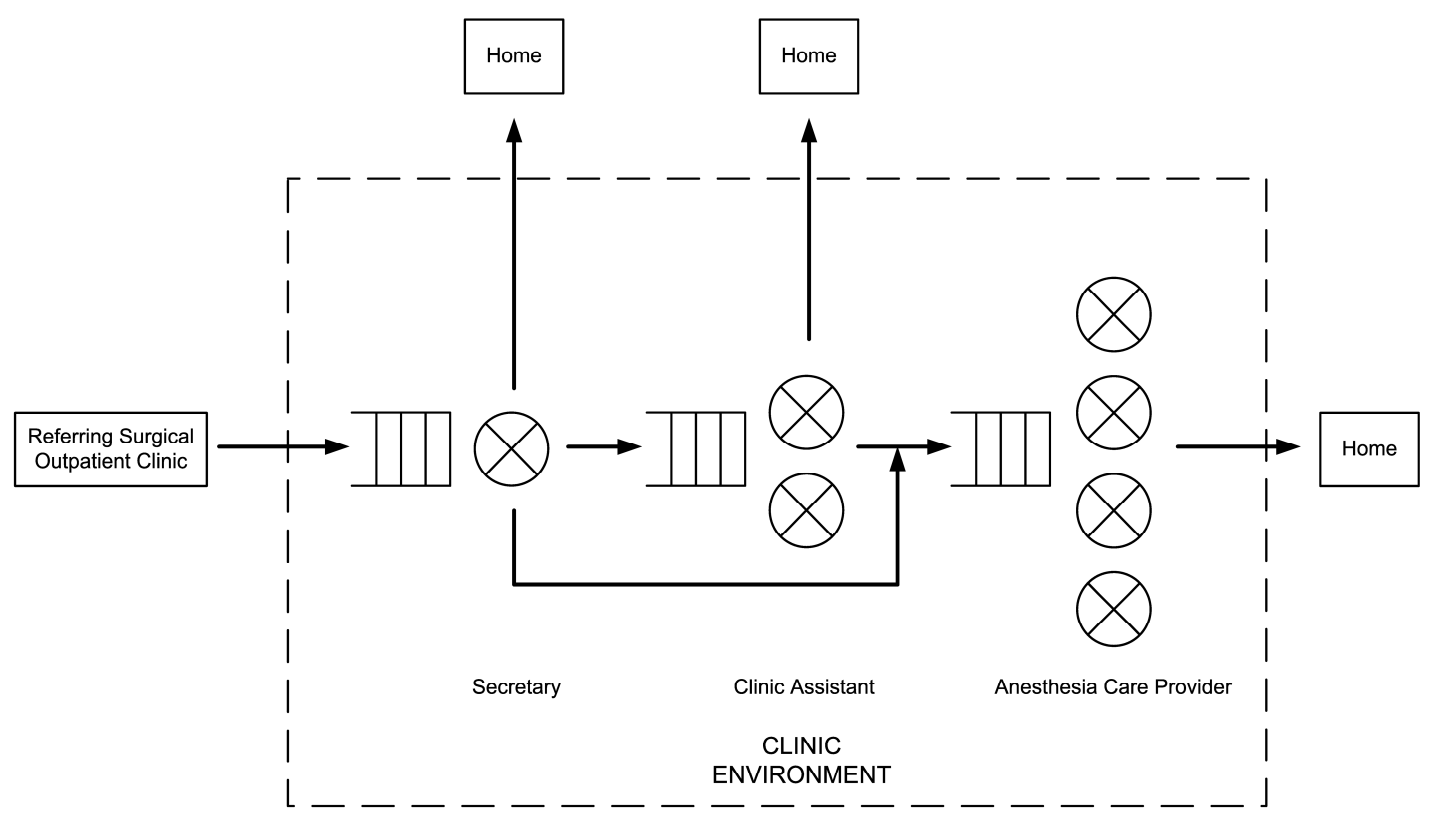

Figure 2 